\title{
Intrauterine Programming of Cardiovascular Diseases in Maternal Diabetes
}

\author{
Romina Higa ${ }^{1,2 *}$, María Laura Leonardi ${ }^{1,2}$ and Alicia Jawerbaum ${ }^{1,2}$ \\ ${ }^{1}$ Facultad de Medicina, Universidad de Buenos Aires, Buenos Aires, Argentina, ${ }^{2}$ Laboratory of Reproduction and \\ Metabolism, CONICET-Universidad de Buenos Aires, CEFYBO, Buenos Aires, Argentina
}

\section{OPEN ACCESS}

Edited by:

Carlos Alonso Escudero, University of the Bío Bío, Chile

Reviewed by: Julienne Rutherford, University of lllinois at Chicago, United States

Helen Jones,

Cincinnati Children's Hospital Medical Center, United States

*Correspondence: Romina Higa rominahiga@gmail.com

Specialty section: This article was submitted to Developmental Physiology, a section of the journal

Frontiers in Physiology

Received: 17 August 2021 Accepted: 11 October 2021 Published: 03 November 2021

Citation:

Higa $R$, Leonardi ML and Jawerbaum A (2021) Intrauterine Programming of Cardiovascular Diseases in Maternal Diabetes.

Front. Physiol. 12:760251. doi: 10.3389/fphys.2021.760251
Maternal diabetes is a prevalent pathology that increases the risk of cardiovascular diseases in the offspring, the heart being one of the main target organs affected from the fetal stage until the adult life. Metabolic, pro-oxidant, and proinflammatory alterations in the fetal heart constitute the first steps in the adverse fetal programming of cardiovascular disease in the context of maternal diabetes. This review discusses both human and experimental studies addressing putative mechanisms involved in this fetal programming of heart damage in maternal diabetes. These include cardiac epigenetic changes, alterations in cardiac carbohydrate and lipid metabolism, damaging effects caused by a pro-oxidant and proinflammatory environment, alterations in the cardiac extracellular matrix remodeling, and specific signaling pathways. Putative actions to prevent cardiovascular impairments in the offspring of mothers with diabetes are also discussed.

Keywords: heart, offspring, animal model, humans, diabetes mellitus, intrauterine programming, pathways

\section{INTRODUCTION}

Cardiovascular diseases are increasing at alarming rates in both developed and developing countries (Balakumar et al., 2016; Bhatnagar et al., 2016). Although lifestyle choices and genetic predisposition are the main contributors to cardiometabolic diseases, growing evidence indicates that in utero exposure to adverse environmental conditions leads the developing offspring to have numerous risk factors, which may have an impact later in life. The concept of developmental programming was first introduced more than 20 years ago by Dr. David Barker, who investigated the association between low birth weight and increased risk of coronary disease in adult life (Barker, 1998). This phenomenon describes the process by which a stimulus or insult during critical periods of growth and development has lasting effects on the structure or function of tissues and organ systems, which will in turn influence changes in body structure and function permanently. Programming occurs because there is a deregulation of the endocrine or metabolic function, or a failure in the development, growth, or interaction of tissues and organ systems of the body. These alterations involve disruptions in gene expression, cell differentiation, proliferation, communication, and/or signaling during critical periods of fetal life and infancy caused by adverse environmental influences. In particular, the risk of fetal programming of metabolic and cardiovascular diseases is well-known to be increased by diabetes in pregnancy (Pandey et al., 2015; Lohse et al., 2018). 
Diabetes can be diagnosed before pregnancy (pregestational type 1 or type 2 diabetes) or have its onset during pregnancy (gestational diabetes mellitus, GDM). All types of diabetes have been shown to induce an adverse fetal programming (Poston, 2010). Epidemiological studies performed in both developed and developing countries have shown an increase in the prevalence and incidence of type 2 diabetes and GDM over the years (Trujillo et al., 2015; Zhu and Zhang, 2016; Hills et al., 2018; Najafi et al., 2019; Gorban de Lapertosa et al., 2021). This review provides information on studies of both human and experimental models addressing putative mechanisms involved in the fetal programming of heart damage in maternal diabetes. Shedding light on these mechanisms would provide tools for future studies in the prevention of cardiovascular disease in offspring exposed to maternal diabetes.

\section{HUMAN STUDIES}

Several studies have shown that children and adolescents exposed to maternal diabetes in utero have significantly higher systolic and mean arterial blood pressures (Cho et al., 2000; Lu et al., 2019; Pathirana et al., 2019). In addition, a correlation has been found between GDM and the rate of cardiovascular disease-related hospitalizations of the offspring up to 18 years (Leybovitz-Haleluya et al., 2018). A recent study has shown that fetuses from diabetic gestation present an increase in the left ventricular mass and wall thickness, which persist into late infancy (Do et al., 2019). The follow up of this longitudinal study in childhood has shown that these alterations persist, with the offspring from diabetic mothers showing higher aortic and ventricular stiffness (Do et al., 2021). Further, a 40-year follow-up study in Denmark has shown that offspring of mothers with diabetes have $29 \%$ increased overall rate of early onset of cardiovascular heart disease (heart failure, hypertensive disease, deep vein thrombosis, and pulmonary embolism; Yu et al., 2019).

Cardiovascular diseases associated with inflammatory processes, as the ones related to diabetes, show increased endothelial cell signal transductions, which favor leukocyte migration by endothelial cell adhesion molecules (Endemann and Schiffrin, 2004). Among these molecules, vascular cell adhesion molecule-1 (VCAM-1) plays important roles in the embryonic development of the cardiovascular system and in cardiovascular diseases (Cook-Mills et al., 2011). In this regards, it has been found that children born to mothers

Abbreviations: VCAM-1, Vascular cell adhesion molecule-1; GDM, Gestational diabetes mellitus; HUVEC, Human umbilical vein endothelial cells; EZH2 $\beta$, Enhancer of zester homolog $2-\beta$; STZ, Streptozotocin; Hspala, Heat shock protein 1a1; NPR1, Natriuretic Peptide Receptor 1; PANK1, Panthothenate kinase; SCAND1, SCAN domain-containing protein 1; Sirt1, Silent information regulator 1; FoxO1, Forkhead box protein O1; FGF, Fibroblast growth factor; GSK3 $\beta$, Glycogen synthase kinase $3 \beta$; PGC1 $\alpha$, Peroxisome proliferator-activated receptor gamma coactivator alpha; mTOR, Mechanistic target of rapamycin; SGK1, Serumand glucocorticoid-inducible kinase 1; PPAR, Peroxisome proliferator activated receptor; NOX, NADPH oxidases; TBARS, Thiobarbituric acid reactive substances; ECM, Extracellular matrix; TGF $\beta-1$, Transforming growth factor $\beta$-1; CTGF, Connective tissue growth factor. with diabetes during pregnancy have increased levels of VCAM-1 and other markers of endothelial activation like E-selectin (Manderson et al., 2002; West et al., 2011). A study of 21 healthy non-Hispanic White children between 8 and 12 years old exposed to GDM in utero reported that, in addition to higher levels of VCAM-1, these children showed an increase in cardiometabolic risk factors, including higher body mass index $z$ scores, higher waist circumference and higher levels of triglycerides, low-density lipoprotein-cholesterol, and leptin compared to unexposed children (West et al., 2011). The latter is important because increased levels of leptin may stimulate oxidative stress, inflammatory reactions, atherogenesis, and thrombosis and thus promote endothelial dysfunction, arterial stiffness, and development and vulnerability of atherosclerotic plaques (Katsiki et al., 2018).

Regarding proposed mechanisms of programming of cardiovascular alterations by maternal diabetes, the main protagonist is epigenetics. Epigenetics can be defined as heritable changes that modify gene expression without altering the DNA sequence itself. Epigenomes are sensitive to their environment and are affected by specific environmental cues, being a plausible mechanism that conditions developmental origin of health and disease in later life. Epigenetic responses during critical windows of fetal development alter gene expression in specific organs or tissues, conditioning their developing and immature functionality and resulting in changes in mature tissues and organs. These responses can either protect the organism from or predispose the organism to disease development in later life. Epigenetic modifications include DNA methylation. DNA methylation involves the covalent addition of a methyl group to the cytosine base in CpG dinucleotide islands of regulatory sites of gene promoter regions that regulate gene expression. This pattern of DNA methylation is highly susceptible to abnormal modifications during adverse gestation and neonatal development (Zhou et al., 2011). In this regard, a genomewide methylation analysis performed in DNA obtained from peripheral blood mononuclear cells from healthy non-Hispanic White children exposed to maternal GDM during intrauterine life showed 84 genes with differentially methylated regions (West et al., 2013). This study also showed that several of the top 10 genes ranked by statistical significance, such as Natriuretic Peptide Receptor 1 (NPR1; related to blood pressure homeostasis), panthothenate kinase (PANK1; a critical enzyme in the synthesis of coenzyme A), SCAN domain-containing protein 1 (SCAND1; which encodes a cofactor that interacts with transcription factor regulators of genes involved in lipid metabolism), and GJA4 (which encodes connexin 37, a component of gap junction channels involved in intercellular communication) were associated with cardiovascular risk (West et al., 2013). Alterations in these genes have been found to be related to hypertensive diseases, altered glucose and lipid metabolism, and myocardial physiology and may thus be related to adverse programming of cardiovascular disease in offspring exposed to maternal diabetes (Babb and Bowen, 2003; Pitzalis et al., 2003; Leu et al., 2011).

Other molecular epigenetic mechanisms that may play a key role in epigenetic inheritance and aberrant development of cardiovascular disease in later life include histone modifications 
and small non-coding RNAs, including microRNAs (also called miRNAs; Watson et al., 2019).

For example, a study of whole peripheral blood from children aged 3-11 years descending from GDM pregnancies showed that these children present an altered miRNA expression profile (Hromadnikova et al., 2020). The study also showed that a large group of the genes affected by the altered miRNA expression is involved in ontologies of diabetes/cardiovascular/ cerebrovascular diseases (Hromadnikova et al., 2020). Moreover, clinical examination of these children indicated that they present an increased incidence of valve problems or heart defects (Hromadnikova et al., 2020).

Studies in human umbilical vein endothelial cells (HUVECs) are an excellent model for the study of vascular endothelium properties and the main biological pathways involved in endothelium function.

Studies in primary culture of HUVECs and human placental microvascular endothelial cells from GDM pregnancies have shown the dysregulation of adenosine metabolism (Westermeier et al., 2011, 2015). Adenosine metabolism, which plays an important role in the methylation cycle, is a proposed mechanism of epigenetic regulation in endothelial cells involved in the cardio-metabolic fetal programming by GDM (Silva et al., 2020).

Another study performed in HUVECs from GDM pregnancies reported a reduction in the expression of histone methyltransferase enhancer of zester homolog $2-\beta(E Z H 2 \beta)$ related to an increase in the expression of miR-101, a microRNA that targets EZH2 $\beta$ (Kuzmichev et al., 2002). EZH2 $\beta$ is part of a multisubunit complex that initiates and maintains the trimethylation of histone $\mathrm{H} 3$ on lysine 27 (H3K27me3), an epigenetic mark associated with heterochromatin formation and gene silencing (Cao et al., 2002), and thus probably related to the regulation of this epigenetic mark in endothelial cells from GDM offspring.

The knowledge regarding the mechanisms of programming of cardiovascular disease in offspring from human patients with diabetes is limited because of the difficulty in performing human intervention studies. Thus, the studies of maternal diabetes in animal models are relevant as they allow us to better understand the developmental origins of disease, moving our understanding from associative studies to mechanistic insights into disease causation.

\section{ANIMAL STUDIES}

Among animal studies, those performed in rodent models are useful because the order and timing of fetal organ development are similar to those in humans. Moreover, the ability to control various aspects of pregnancy and the pre- and post-natal environment makes rodent models as attractive experimental systems to examine how maternal diabetes during pregnancy programs the physiology of organ systems and increases the risk of cardiometabolic disease in the offspring.

Experimental models of diabetes in pregnancy can have a genetic origin or be obtained by chemical induction with drugs such as streptozotocin (STZ), which, at the appropriate dose, acts by selectively destroying pancreatic cells, leading to insulin deficiency and hyperglycemia in different animals (Jawerbaum and White, 2010).

Regarding the mechanisms underlying the programming of cardiovascular diseases, studies in animal models can be divided into those focused on (1) epigenetic mechanisms, (2) altered pathways of cellular metabolism, (3) alterations induced by a pro-oxidant/proinflammatory environment, and (4) alterations in extracellular matrix remodeling and intercellular communication.

\section{Animal Studies Focused on Cardiovascular Epigenetic Mechanisms}

Animal studies focused on epigenetic mechanisms may improve our understanding of how epigenetic gene transcriptional regulation responds to an altered gestational milieu to influence the developmental origin of health and disease.

As an example, a study of the neonatal heart in a mouse model of diabetes showed a 10-fold increase in the DNA methylation of gene promoter regions of many important cardiac genes (Lister et al., 2013). In another study, rat neonatal hearts exposed to maternal diabetes showed altered gene-activating (H3Ac, H3K4me3) and gene-suppressive (H3K27me3) histone marks (Upadhyaya et al., 2017). In this study, chromatinimmunoprecipitation-sequencing and bioinformatics identified that the promoters of two functionally related genes, heat shock protein $1 a 1$ (Hspa1a) and Hspa1b, showed enriched H3K4me3 peaks (Upadhyaya et al., 2017). Hspala has been proposed as an independent prognostic marker of heart failure (Jenei et al., 2013). This suggests that Hspala may be a relevant marker linking maternal diabetes and offspring heart failure.

In a rat model of diabetes in pregnancy induced by STZ administration on day 12 of gestation, cardiomyocytes from 6-week-old offspring showed an increase in global DNA methylation status, together with an increase in the cardiac expression of DNA methyltransferase 3A and reduced expression of the silent information regulator 1 (Sirt1), a class III histone deacetylase (Chen et al., 2019). This altered expression of Sirtl was reversed ex vivo with a DNA-methylation inhibitor and in vivo with the antioxidant $\mathrm{N}$-acetyl-cysteine, suggesting that Sirt1 expression in the heart of offspring from dams with diabetes is epigenetically regulated and affected by an oxidative environment (Chen et al., 2019). Sirt1 functions as a regulator of acetylation, which is important to maintain cardiac mitochondrial integrity and normal myocardium development (Planavila et al., 2012). One of Sirt1 targets genes is the transcription factor forkhead box protein $\mathrm{O} 1$ (FoxO1), which, in the cardiovascular system, participates in myocardial metabolic stress adaptation, oxidative stress, endothelial dysfunction, and other processes related to inflammation and apoptosis (Kandula et al., 2016). FoxO1 deacetylation by Sirt1 induces the attenuation of its ability to bind DNA and favors its phosphorylation and consequently its inactivation. In line with this, in a rat model of mild diabetes, the heart of male adult offspring was found to show an increase in active FoxO1 (Musikant et al., 2019). 
Another putative epigenetic mechanism involved in the programming of cardiovascular diseases involves biogenesis of miRNAs which can be delivered by exosomes. Exosomes are nanovesicles that contain intact and functional mRNAs, miRNAs and proteins, which function as pivotal mediators of cell-cell communication among neighboring or distant cells and play a key role in multiple physiological or pathological processes (Greening et al., 2017).

It has been demonstrated that, during pregnancy, both fetomaternal and maternal-fetal exosomal trafficking can occur, and that exosomes are able to cross placental barriers and reach fetal tissues (Sheller-Miller et al., 2019; Czernek and Düchler, 2020). Although the precise mechanism has not yet been completely clarified, in vitro studies have shown that endothelial cells absorb placental-derived exosomes through endocytosis (Tong et al., 2017). A study by Shi et al. of serum exosomes isolated from pregnant mice with STZ-induced diabetes showed that these vesicles present differences in the expression of more than 200 miRNAs (Shi et al., 2017), some of which, including miR-133, miR-30, miR-99, and miR-23, are involved in cardiac development and cardiovascular diseases (Bang et al., 2012; Izarra et al., 2017; Yin and Yang, 2019). These authors also found that serum exosomes isolated from these diabetic pregnant mice, fluorescently labeled for tracking and then injected into normal pregnant mice via the tail vein, were able to cross the placental barrier and infiltrate into embryonic tissues, including the heart (Shi et al., 2017). Finally, these authors showed that the injections of these exosomes increased embryonic heart malformations and induced alterations in the cardiac systolic function in the morphologically normal fetus (Shi et al., 2017), highlighting the role of maternal exosome-derived microRNAs in the induction of fetal cardiovascular defects.

\section{Animal Studies Focused on Altered Pathways of Cardiac Cellular Metabolism}

Key pathways of cellular metabolism have been shown to be altered in cardiac cells of offspring from animal models of diabetes. A transcriptomic study performed in neonatal heart from dams treated with a combination of high-fat diet and STZ at day 14 of pregnancy, showed many changes in key genes of metabolic cardiac pathways. Among them, a downregulation of fibroblast growth factor (FGF), which in turn downregulates PI3K/AKT pathway activation that lead to increased glycogen synthase kinase $3 \beta$ (GSK3 $\beta$ ) and peroxisome proliferator-activated receptor gamma coactivator alpha (PGC1 $\alpha$; Preston et al., 2020). PI3K/AKT is an insulin sensitive pathway that modulates metabolic pathways as gluconeogenesis (modulated by GSK3 $\beta$ ) and mitochondrial function and biogenesis (modulated by PGC1 $\alpha$ ), between others. These changes would contribute to an increased translation of mitochondrial proteins, mitochondrial biogenesis, and gluconeogenesis.

Another important pathway of cellular metabolism is regulated by the mechanistic target of rapamycin (mTOR), a cellular sensor for energy metabolism and nutrient availability that controls cellular growth and metabolism. mTOR forms two different complexes (mTORC1 and mTORC2), which differ in downstream signaling pathways and function (Saxton and Sabatini, 2017). Activated mTORC1 plays a central role in the control of cell growth and proliferation, while mTORC2 plays a key role in cell survival, metabolism, proliferation, and cytoskeleton organization (Saxton and Sabatini, 2017). In a study of a rat model of STZ-induced mild diabetes, the heart of adult offspring showed a reduction in mTOR protein levels and in the phosphorylation of serum- and glucocorticoid-inducible kinase 1 (SGK1), a downstream target of the mTORC2 pathway, which phosphorylates FoxO1 (Musikant et al., 2019). Phosphorylation of the FoxO family by SGK1 induces its nuclear exclusion and inactivation, providing another possible mechanism explaining FoxO1 increased activity (Brunet et al., 2001; Musikant et al., 2019). Interestingly, in a study in which a diet enriched in fish oil was administered to dams with STZ-induced diabetes, the offspring's neonatal heart showed an increase in the reduced phosphorylation of mTOR and AKT, both in Ser-473 (phosphorylation induced by mTORC2) and in Thr-308 (phosphorylation activated by growth factors pathways; Nasu-Kawaharada et al., 2013).

In the offspring heart of an obesity model with hyperglycemia and hyperinsulinemia, Zhang et al. found that the mTORC1 signaling pathway was increased. These authors also found that a maternal treatment with the antioxidant $\mathrm{N}$-acetyl-cysteine during pregnancy normalized the altered mTOR signaling in the offspring heart (Zhang et al., 2021).

Regarding lipid metabolism, in maternal diabetes, insulin concentration, and/or signaling deficiency may lead to an excess of metabolic substrates and impairments in maternal lipid metabolism. In addition, diabetes in pregnancy frequently induces dyslipemia in the mother (Herrera and Ortega-Senovilla, 2010). We have previously reviewed the importance of lipids in the programming of metabolic and cardiovascular alterations in the offspring (Higa and Jawerbaum, 2013).

In an experimental model of high-fat and high-cholesterol diet during pregnancy and lactation, which induces dyslipemia, glucose intolerance, and reduced insulin sensitivity, male offspring showed increased blood pressure, impaired vascular reactivity, and lower response to endothelium-dependent vasorelaxation (Guimarães et al., 2020). Interestingly, in that study, an administration of a specific strain of the probiotic Lactiplantibacillus plantarum improved the lipid profile and insulin resistance and restored the dysbiotic gut microbiota in dams, reducing blood pressure and recovering the vascular function in the adult offspring (Guimarães et al., 2020). In another study in a STZ-induced experimental model of mild diabetes, 5-month-old offspring showed increased glycemia, insulinemia, and triglyceridemia (Capobianco et al., 2015). Besides, a sex-dependent lipid accumulation was observed in their heart as female offspring showed increased levels of free fatty acids, cholesterol, and phospholipids and male offspring showed increased levels of triglycerides (Capobianco et al., 2015). These results are relevant because an increase in lipid accumulation in the heart has been related to impaired mitochondrial metabolism and dynamics and increased oxidative stress, cardiomyocyte apoptosis, myocardial fibrosis, and contractile dysfunction (McGavock et al., 2006; Wende and Abel, 2010; Elezaby et al., 2015). Although the mechanisms 
of sex-dependent lipid overaccumulation in the heart are unknown, they may be due to changes in lipid metabolism, which are in turn dependent on multiple factors, including the sex-dependent impact of hormones, mitochondrial function, and genetic and epigenetic differences (Gabory et al., 2009; Vijay et al., 2015; Regitz-Zagrosek and Kararigas, 2017).

The intrauterine programming of cardiovascular impairments and the regulation of peroxisome proliferator activated receptor (PPAR)-dependent pathways have also been found to be sex-dependent (Benz et al., 2012; Regitz-Zagrosek and Kararigas, 2017). In experimental models of STZ-induced diabetes, PPAR $\alpha$, a nuclear receptor highly involved in heart metabolic processes and lipid metabolism, has been found increased in the heart of both males and females at the neonatal stage but only in that of males at the prepubertal stage (Higa et al., 2017). An increased level of PPAR $\alpha$ is a marker of metabolic substrate utilization as this nuclear receptor regulates multiple genes of lipid catabolism (Lefebvre et al., 2006). Oleic acid, present at high concentrations in olive oil, is a natural agonist that interacts with the ligandbinding domain of the PPAR and leads to its activation (Xu et al., 1999). Interestingly, a maternal olive oil-supplemented diet administered during diabetic rat pregnancy prevents lipid overaccumulation in the offspring's heart, probably as a consequence of improving intrauterine metabolic homeostasis (Capobianco et al., 2015). The PPAR $\alpha$ gene was susceptible to epigenetic modification in the livers of offspring of dams fed a low-protein diet (Lillycrop et al., 2005) and PPAR expression in the livers of offspring from an animal model of gestational diabetes was found to be modulated by different miRNAs (Fornes et al., 2018). However, further research in needed to elucidate whether maternal diabetes also induces epigenetic modifications of the PPAR $\alpha$ gene in the offspring's heart.

In an animal model of diabetes induced by STZ administration during pregnancy, results showed diastolic dysfunction in the offspring at the neonatal stage (Mdaki et al., 2016) and impaired mitochondrial dynamics in neonatal cardiomyocytes (Larsen et al., 2019). The term "mitochondrial dynamics" refers to the coordinated cycles of fission and fusion occurring in these organelles, which are important to maintain their shape, distribution and size. Mitochondrial dynamics plays an important role in both cardiac development and long-term heart health (Dorn et al., 2015). Interestingly, a study performed in cardiomyocytes of neonates from rats with diabetes showed $50 \%$ reduction in fusion events and $30 \%$ reduction in fission events, as well as a pro-fission imbalance in the ratio of these events and a higher number of fragmented mitochondrial morphology (Larsen et al., 2019).

Alterations in mitochondrial function lead to an increase in reactive oxygen species and thus to an increase in oxidative stress (Peoples et al., 2019), which in turn plays a key role in the programming of cardiovascular alterations (Gupta et al., 2004; Rueda-Clausen et al., 2012).

\section{Animal Studies Focused on Cardiovascular Alterations Induced by a Pro-oxidant/ Proinflammatory Environment}

In cardiac tissues, a major source of reactive oxygen species under both physiological and pathological conditions is NADPH oxidases (NOX; Lassegue et al., 2012). In a study performed in a rat model of diabetes in pregnancy induced by STZ administration on day 12 of gestation, the heart of the 6-weekold offspring showed an increase in NOX1 and NOX2 together with an increase in reactive oxygen species in the left ventricle (Chen et al., 2019), and the heart of adult offspring still showed an increase in NOX2 expression (Zhang et al., 2018). Maternal treatment with $\mathrm{N}$-acetyl-cysteine prevents the increased NOX2 transcript levels in the offspring heart from a model of obesity and diabetes (Zhang et al., 2021).

In another study in a model of mild diabetes obtained by STZ administration, the offspring's heart at neonatal stage showed an increase in nitrated proteins, which evidences protein damage by peroxynitrites (Higa et al., 2017), potent oxidant agents generated as the product of nitric oxide and superoxide anion reaction. In that study, neonatal glycemia of offspring exposed or not to maternal diabetes was similar, indicating that peroxynitrite-induced damage in the offspring's heart occurs in the absence of alterations in neonatal glycemia. However, at a prepubertal stage, glycemia was increased only in male offspring from rats with diabetes (Higa et al., 2017).

Lipids can also be the target of reactive oxygen species. In an experimental model of STZ-induced diabetes, sex-dependent differences were found related to lipoperoxidation, as only neonatal hearts of male offspring showed an increase in thiobarbituric acid reactive substances (TBARS), which are formed as a byproduct of lipid peroxidation (Higa et al., 2017). At the fetal stage, lipoperoxidation has also been observed only in the hearts of male fetuses, suggesting changes during fetal development that persist into adult life (Kurtz et al., 2014).

Regarding maternal treatments that have an impact in modulating the pro-oxidant intrauterine environment, at our lab, we observed that a maternal treatment with an olive oil-supplemented diet administered during pregnancy was able to prevent the increased pro-oxidant markers as well as apoptosis in the heart of 21-day-old offspring of diabetic rats (Roberti et al., 2020). At our laboratory, we have also evaluated a maternal treatment with the mitochondrial antioxidant idebenone in a model of mild diabetes during pregnancy to address the role of oxidative stress and mitochondrial dysfunction in the programming of cardiac alterations and observed that this treatment prevents the increase in markers of oxidative damage in the heart of offspring from diabetic rats at a prepubertal stage (Higa et al., 2017). These results highlight the relevance of mitochondrialrelated reactive oxygen species in the programming of heart alterations by maternal diabetes.

\section{Animal Studies Focused on Alterations in the Cardiac Extracellular Matrix and Intercellular Communication}

The cardiac extracellular matrix (ECM) is a complex and dynamic structure that forms a three-dimensional network in the cardiac interstitium. It provides structural support for several distinct cell types, contains growth factors and cellular adhesion proteins, and integrates extracellular signals and 
cellular responses (Frangogiannis, 2017). In cardiac pathological conditions, ECM synthesis and chemical composition are subject to changes under different environmental stimuli that distort the architecture of the matrix network, modulating the proliferation, migration and activation of cardiac fibroblasts, thus playing a major role in the development of cardiac diseases (Li et al., 2018). During organogenesis, mouse embryo cultures in hyperglycemic conditions have been found to show an increased expression of cardiac fibronectin and transforming growth factor $\beta$-1 (TGF $\beta$-1; Smoak, 2004), a fibrogenic growth factor that mediates ECM remodeling. In the male offspring heart from a mouse model of obesity and diabetes, Zhang et al. found an increased expression of TGF $\beta-1$ together with myocardial fibrosis and left ventricular structural alterations. These authors also found that all these programmed alterations were prevented by a maternal treatment with $\mathrm{N}$-acetyl-cysteine during pregnancy (Zhang et al., 2021). Several downstream actions of TGF- $\beta 1$ can be mediated by connective tissue growth factor (CTGF), a secreted multifunctional protein also involved in the regulation of ECM deposition during development as well as during pathological conditions (Frangogiannis, 2012). A study in an animal model of STZ-induced mild diabetes showed an increase in CTGF levels in the hearts of neonates (Higa et al., 2017). In the heart, CTGF has been found to be epigenetically regulated by microRNA-133 and -30 (Duisters et al., 2009), pointing to these miRNAs as putative epigenetic marks that may be involved in the intrauterine programming of CTGF upregulation in the offspring's heart as they have also been found increased in serum exosomes isolated from pregnant dams with diabetes (Shi et al., 2017).

The balance between ECM synthesis and degradation is of crucial relevance in maintaining cardiac structural integrity. In this context, it is important to mention the role of matrix metalloproteinases (MMPs), which are proteolytic enzymes able to degrade ECM components. Expression and/or activity of MMPs are upregulated by the pro-oxidant/proinflammatory environment. In particular, MMP-9 expression has been found

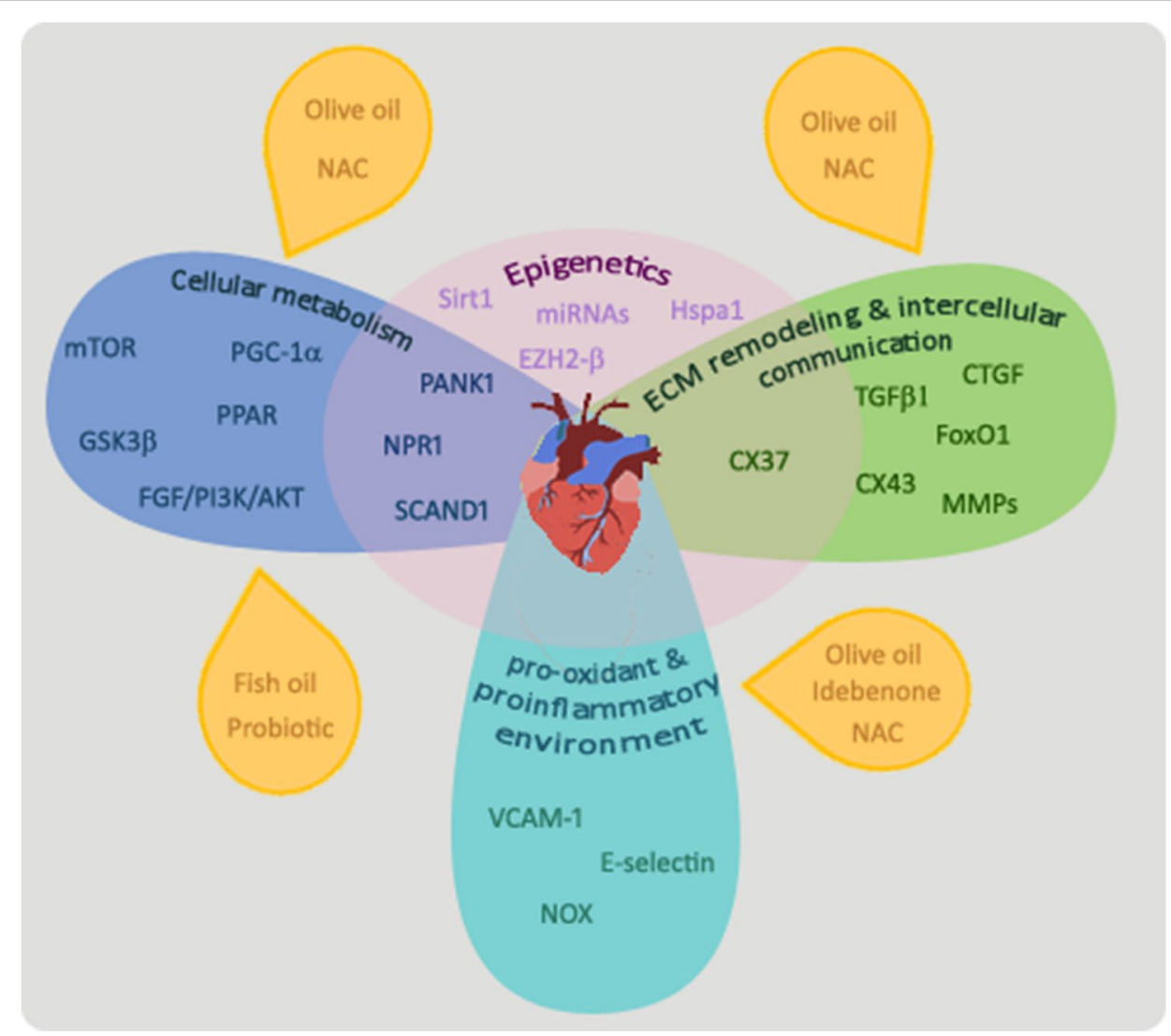

FIGURE 1 | Mechanisms involved in the programming of cardiovascular alterations in offspring by maternal diabetes. Main players that regulate cellular metabolism in the heart of offspring exposed to diabetes in utero are mTOR, PPAR, PANK1, PGC1 $\alpha$, GDK3 $\beta$, NPR1, SCAND1, and the FGF/PI3K/AKT pathway. Maternal treatments with probiotics or a diet enriched in olive or fish oil or $\mathrm{N}$-acetyl-cysteine have been shown to ameliorate cardiac cell metabolism of offspring from diabetic dams. TGF $\beta$-1, CTGF, FoxO1, matrix metalloproteinases (MMPs), and connexin 37 (CX37) and 43 (CX43) have been shown to play important roles in the process of ECM remodeling and intercellular communication. A maternal treatment with $\mathrm{N}$-acetyl-cysteine (NAC) or with an olive oil-supplemented diet was able to prevent alterations in the ECM remodeling process observed in the offspring's heart of animal models of diabetes. An increased pro-oxidant/proinflammatory process and related molecules (VCAM-1, E-selectin, and NOX) have been shown to be involved in the cardiovascular alterations programmed by maternal diabetes. Maternal treatments with an olive oil-supplemented diet or with antioxidants, as idebenone or $\mathrm{N}$-acetyl-cysteine have beneficial effects on this process. All these pathways are interconnected and the underlying mechanism affecting them may be epigenetic phenomena. 
increased in hearts of neonates from diabetic rats (Higa et al., 2017). Interestingly, alterations in these markers of ECM remodeling persist and are sex-dependent at a prepubertal stage of development. At 21 days old, only males show an increased expression of MMP-9, possibly related to increased markers of pro-oxidant/proinflammatory processes, which are observed only in the heart of male fetuses and 21-day-old male offspring (Kurtz et al., 2014; Higa et al., 2017; Roberti et al., 2020). Indeed, the stimulation of several fibrogenic pathways by diabetes is related to the generation of reactive oxygen species and the induction of secretion of pro-inflammatory cytokines and chemokines (Tuleta and Frangogiannis, 2021). Moreover, maternal treatment with an olive oil-supplemented diet administered during pregnancy, which has been found to prevent cardiac pro-oxidant/ proinflammatory processes in the offspring, is also able to prevent the increased expression of CTGF and deposition of collagen IV and fibronectin in the heart of 21-day-old offspring of diabetic rats (Roberti et al., 2020).

FoxO1 is a TGF $\beta-1$ downstream crucial player in cardiac fibroblast conversion into cardiac myofibroblasts, which, under pathological conditions such as diabetes mellitus, synthesizes and secretes high amounts of ECM proteins (Vivar et al., 2021). Male adult offspring from rats with mild diabetes show an increase in cardiac active FoxO1, together with an increase in the mRNA levels of its target genes in the heart, Mmp-2 and Ctgf, and in collagen deposition and fibrosis (Musikant et al., 2019). These offspring's hearts also show reduced expression of connexin 43, a target of matrix metalloproteinase 2 and main component of the gap junctions and hemichannels in myocytes (Musikant et al., 2019). As connexin 43 is a key factor in electrical coupling and its altered expression alters normal impulse propagation (van Rijen et al., 2006), its reduced levels in the offspring's heart is likely related to cardiac dysfunction. These alterations were found concomitantly with increased markers of cardiomyopathy and increased cardiovascular risk factors as glycemia, triglyceridemia, and insulinemia (Musikant et al., 2019). Together, these results suggest an important role of FoxO1 activation in the cardiac alterations related to cardiac ECM remodeling induced by intrauterine programming in maternal diabetes (Musikant et al., 2019).

\section{REFERENCES}

Babb, R., and Bowen, B. R. (2003). SDP1 is a peroxisome-proliferator-activated receptor gamma 2 co-activator that binds through its SCAN domain. Biochem. J. 370, 719-727. doi: 10.1042/bj20021378

Balakumar, P., Maung-U, K., and Jagadeesh, G. (2016). Prevalence and prevention of cardiovascular disease and diabetes mellitus. Pharmacol. Res. 113, 600-609. doi: $10.1016 /$ j.phrs.2016.09.040

Bang, C., Fiedler, J., and Thum, T. (2012). Cardiovascular importance of the microRNA-23/27/24 family. Microcirculation 19, 208-214. doi: 10.1111/j.1549-8719.2011.00153.x

Barker, D. J. P. (1998). Mothers, Babies, and Health in Later Life. Churchill Livingstone.

Benz, V., Kintscher, U., and Foryst-Ludwig, A. (2012). Sex-specific differences in type 2 diabetes mellitus and dyslipidemia therapy: PPAR agonists. Handb. Exp. Pharmacol. 214, 387-410. doi: 10.1007/978-3-642-30726-3_18

\section{CONCLUSION}

The mechanisms involved in fetal programming of heart damage in maternal diabetes reviewed in this study include the dysregulation of key players in cardiac cell metabolism, increased damaging effects of a pro-oxidant and proinflammatory environment, and alterations in the cardiac ECM remodeling and intercellular communication. As summarized in Figure 1, all these mechanisms and the main players that regulate these altered pathways are interconnected and differentially modulated by epigenetic modifications. This evidences the complex and multiple pathways able to induce programming of cardiovascular diseases in the offspring of diabetic dams, but also, due to the close interaction of these main pathways, brings opportunities to facilitate intervention to provide protective effects. Dietary treatments that improve the maternal metabolism altered by diabetes, such as those with probiotics or supplementation with olive or fish oil, have been shown to have beneficial effects in the cardiac cell metabolism of the offspring. Some of the studies reviewed in this work point to olive oil supplementation in the maternal diet and the maternal treatment with $\mathrm{N}$-acetyl-cysteine as effective treatments able not only to improve cardiac cell metabolism but also to reverse the programmed damaging effect of the pro-oxidant and proinflammatory environment and the alterations in the cardiac ECM remodeling in the offspring heart. Further research addressing plausible treatments in animal models of maternal diabetes and their translation into clinical practice is encouraged.

\section{AUTHOR CONTRIBUTIONS}

$\mathrm{RH}$ and $\mathrm{AJ}$ designed the research. $\mathrm{RH}$ and $\mathrm{ML}$ conducted the research. RH wrote the manuscript. AJ contributed to the critical reading of the manuscript. All authors contributed to the article and approved the submitted version.

\section{FUNDING}

This work was supported by the Agencia Nacional de Promoción Científica y Tecnológica de Argentina (PICT 2015130 and PIDC 2015 0064).
Bhatnagar, P., Wickramasinghe, K., Wilkins, E., and Townsend, N. (2016). Trends in the epidemiology of cardiovascular disease in the UK. Heart 102, 1945-1952. doi: 10.1136/heartjnl-2016-309573

Brunet, A., Park, J., Tran, H., Hu, L. S., Hemmings, B. A., and Greenberg, M. E. (2001). Protein kinase SGK mediates survival signals by phosphorylating the forkhead transcription factor FKHRL1 (FOXO3a). Mol. Cell Biol. 21, 952-965. doi: 10.1128/MCB.21.3.952-965.2001

Cao, R., Wang, L., Wang, H., Xia, L., Erdjument-Bromage, H., Tempst, P., et al. (2002). Role of histone H3 lysine 27 methylation in Polycomb-group silencing. Science 298, 1039-1043. doi: 10.1126/science.1076997

Capobianco, E., Pelesson, M., Careaga, V., Fornes, D., Canosa, I., Higa, R., et al. (2015). Intrauterine programming of lipid metabolic alterations in the heart of the offspring of diabetic rats is prevented by maternal diets enriched in olive oil. Mol. Nutr. Food Res. 59, 1997-2007. doi: 10.1002/ mnfr.201500334 
Chen, Z., Gong, L., Zhang, P., Li, Y., Liu, B., Zhang, L., et al. (2019). Epigenetic down-regulation of sirt 1 via DNA methylation and oxidative stress signaling contributes to the gestational diabetes mellitus-induced fetal programming of heart ischemia-sensitive phenotype in late life. Int. J. Biol. Sci. 15, 1240-1251. doi: $10.7150 /$ ijbs.33044

Cho, N. H., Silverman, B. L., Rizzo, T. A., and Metzger, B. E. (2000). Correlations between the intrauterine metabolic environment and blood pressure in adolescent offspring of diabetic mothers. J. Pediatr. 136, 587-592. doi: 10.1067/ mpd.2000.105129

Cook-Mills, J. M., Marchese, M. E., and Abdala-Valencia, H. (2011). Vascular cell adhesion molecule-1 expression and signaling during disease: regulation by reactive oxygen species and antioxidants. Antioxid. Redox Signal. 15, 1607-1638. doi: 10.1089/ars.2010.3522

Czernek, L., and Düchler, M. (2020). Exosomes as messengers between mother and fetus in pregnancy. Int. J. Mol. Sci. 21:4264. doi: 10.3390/ijms21124264

Do, V., Al-Hashmi, H., Ojala, T., Jain, V., Colen, T., Goncalvez-Alvarez, S., et al. (2019). Cardiovascular health of offspring of diabetic mothers from the fetal through late-infancy stages. JACC Cardiovasc. Imaging 12, 932-934. doi: 10.1016/j.jcmg.2018.10.016

Do, V., Eckersley, L., Lin, L., Davidge, S. T., Stickland, M. K., Ojala, T., et al. (2021). Persistent aortic stiffness and left ventricular hypertrophy in children of diabetic mothers. CJC Open 3, 345-353. doi: 10.1016/j.cjco.2020.10.020

Dorn, G. W., Vega, R. B., and Kelly, D. P. (2015). Mitochondrial biogenesis and dynamics in the developing and diseased heart. Genes Dev. 29, 1981-1991. doi: 10.1101/gad.269894.115

Duisters, R. F., Tijsen, A. J., Schroen, B., Leenders, J. J., Lentink, V., van der Made, I., et al. (2009). miR-133 and miR-30 regulate connective tissue growth factor: implications for a role of microRNAs in myocardial matrix remodeling. Circ. Res. 104, 170-178, 6p following 178. doi: 10.1161/ CIRCRESAHA.108.182535

Elezaby, A., Sverdlov, A. L., Tu, V. H., Soni, K., Luptak, I., Qin, F., et al. (2015). Mitochondrial remodeling in mice with cardiomyocyte-specific lipid overload. J. Mol. Cell. Cardiol. 79, 275-283. doi: 10.1016/j.yjmcc.2014.12.001

Endemann, D. H., and Schiffrin, E. L. (2004). Endothelial dysfunction. J. Am. Soc. Nephrol. 15, 1983-1992. doi: 10.1097/01.ASN.0000132474.50966.DA

Fornes, D., White, V., Higa, R., Heinecke, F., Capobianco, E., and Jawerbaum, A. (2018). Sex-dependent changes in lipid metabolism, PPAR pathways and microRNAs that target PPARs in the fetal liver of rats with gestational diabetes. Mol. Cell. Endocrinol. 461, 12-21. doi: 10.1016/j.mce.2017.08.004

Frangogiannis, N. G. (2012). Matricellular proteins in cardiac adaptation and disease. Physiol. Rev. 92, 635-688. doi: 10.1152/physrev.00008.2011

Frangogiannis, N. G. (2017). The extracellular matrix in myocardial injury, repair, and remodeling. J. Clin. Invest. 127, 1600-1612. doi: 10.1172/JCI87491

Gabory, A., Attig, L., and Junien, C. (2009). Sexual dimorphism in environmental epigenetic programming. Mol. Cell. Endocrinol. 304, 8-18. doi: 10.1016/j. mce.2009.02.015

Gorban de Lapertosa, S., Sucani, S., Salzberg, S., Alvariñas, J., Faingold, C., Jawerbaum, A., et al. (2021). Prevalence of gestational diabetes mellitus in Argentina according to the Latin American Diabetes Association (ALAD) and International Association of Diabetes and Pregnancy Study Groups (IADPSG) diagnostic criteria and the associated maternal-neonatal complications. Health Care Women Int. 42, 636-656. doi: 10.1080/07399332.2020.1800012

Greening, D. W., Xu, R., Gopal, S. K., Rai, A., and Simpson, R. J. (2017). Proteomic insights into extracellular vesicle biology - defining exosomes and shed microvesicles. Expert Rev. Proteomics 14, 69-95. doi: 10.1080/14789450.2017.1260450

Guimarães, K. S. d. L., Braga, V. d. A., Noronha, S. I. S. R. d., Costa, W. K. A. d., Makki, K., Cruz, J. d. C., et al. (2020). Lactiplantibacillus plantarum WJL administration during pregnancy and lactation improves lipid profile, insulin sensitivity and gut microbiota diversity in dyslipidemic dams and protects male offspring against cardiovascular dysfunction in later life. Food Funct. 11, 8939-8950. doi: 10.1039/D0FO01718C

Gupta, P., Narang, M., Banerjee, B. D., and Basu, S. (2004). Oxidative stress in term small for gestational age neonates born to undernourished mothers: a case control study. BMC Pediatr. 4:14. doi: 10.1186/1471-2431-4-14

Herrera, E., and Ortega-Senovilla, H. (2010). Disturbances in lipid metabolism in diabetic pregnancy - are these the cause of the problem? Best Pract. Res. Clin. Endocrinol. Metab. 24, 515-525. doi: 10.1016/j.beem.2010.05.006
Higa, R., and Jawerbaum, A. (2013). Intrauterine effects of impaired lipid homeostasis in pregnancy diseases. Curr. Med. Chem. 20, 2338-2350. doi: 10.2174/0929867311320180005

Higa, R., Roberti, S. L., Capobianco, E., Fornes, D., White, V., and Jawerbaum, A. (2017). Pro-oxidant/pro-inflammatory alterations in the offspring's heart of mild diabetic rats are regulated by maternal treatments with a mitochondrial antioxidant. Reprod. Toxicol. 73, 269-279. doi: 10.1016/j.reprotox.2017.06.051

Hills, A. P., Arena, R., Khunti, K., Yajnik, C. S., Jayawardena, R., Henry, C. J., et al. (2018). Epidemiology and determinants of type 2 diabetes in South Asia. Lancet Diabetes Endocrinol. 6, 966-978. doi: 10.1016/S22138587(18)30204-3

Hromadnikova, I., Kotlabova, K., Dvorakova, L., Krofta, L., and Sirc, J. (2020). Substantially altered expression profile of diabetes/cardiovascular/ cerebrovascular disease associated microRNAs in children descending from pregnancy complicated by gestational diabetes mellitus-one of several possible reasons for an increased cardiovascular risk. Cell 9:1557. doi: 10.3390/ cells 9061557

Izarra, A., Moscoso, I., Cañón, S., Carreiro, C., Fondevila, D., Martín-Caballero, J., et al. (2017). miRNA-1 and miRNA-133a are involved in early commitment of pluripotent stem cells and demonstrate antagonistic roles in the regulation of cardiac differentiation. J. Tissue Eng. Regen. Med. 11, 787-799. doi: 10.1002/ term.1977

Jawerbaum, A., and White, V. (2010). Animal models in diabetes and pregnancy. Endocr. Rev. 31, 680-701. doi: 10.1210/er.2009-0038

Jenei, Z. M., Gombos, T., Förhécz, Z., Pozsonyi, Z., Karádi, I., Jánoskuti, L., et al. (2013). Elevated extracellular HSP70 (HSPA1A) level as an independent prognostic marker of mortality in patients with heart failure. Cell Stress Chaperones 18, 809-813. doi: 10.1007/s12192-013-0425-Z

Kandula, V., Kosuru, R., Li, H., Yan, D., Zhu, Q., Lian, Q., et al. (2016). Forkhead box transcription factor 1: role in the pathogenesis of diabetic cardiomyopathy. Cardiovasc. Diabetol. 15, 44. doi: 10.1186/s12933-016-0361-1

Katsiki, N., Mikhailidis, D. P., and Banach, M. (2018). Leptin, cardiovascular diseases and type 2 diabetes mellitus review-article. Acta Pharmacol. Sin. 39, 1176-1188. doi: 10.1038/aps.2018.40

Kurtz, M., Capobianco, E., Martinez, N., Roberti, S. L., Arany, E., and Jawerbaum, A. (2014). PPAR ligands improve impaired metabolic pathways in fetal hearts of diabetic rats. J. Mol. Endocrinol. 53, 237-246. doi: 10.1530/JME-14-0063

Kuzmichev, A., Nishioka, K., Erdjument-Bromage, H., Tempst, P., and Reinberg, D. (2002). Histone methyltransferase activity associated with a human multiprotein complex containing the enhancer of Zeste protein. Genes Dev. 16, 2893-2905. doi: $10.1101 / \operatorname{gad} .1035902$

Larsen, T. D., Sabey, K. H., Knutson, A. J., Gandy, T. C. T., Louwagie, E. J., Lauterboeck, L., et al. (2019). Diabetic pregnancy and maternal high-fat diet impair mitochondrial dynamism in the developing fetal rat heart by sex-specific mechanisms. Int. J. Mol. Sci. 20, 1-20. doi: 10.3390/ijms20123090

Lassegue, B., San Martin, A., and Griendling, K. K. (2012). Biochemistry, physiology, and pathophysiology of NADPH oxidases in the cardiovascular system. Circ. Res. 110, 1364-1390. doi: 10.1161/CIRCRESAHA.111.243972

Lefebvre, P., Chinetti, G., Fruchart, J. C., and Staels, B. (2006). Sorting out the roles of PPAR alpha in energy metabolism and vascular homeostasis. J. Clin. Invest. 116, 571-580. doi: 10.1172/JCI27989

Leu, H.-B., Chung, C.-M., Chuang, S.-Y., Bai, C.-H., Chen, J.-R., Chen, J.-W., et al. (2011). Genetic variants of connexin37 are associated with carotid intima-medial thickness and future onset of ischemic stroke. Atherosclerosis 214, 101-106. doi: 10.1016/j.atherosclerosis.2010.10.010

Leybovitz-Haleluya, N., Wainstock, T., Landau, D., and Sheiner, E. (2018). Maternal gestational diabetes mellitus and the risk of subsequent pediatric cardiovascular diseases of the offspring: a population-based cohort study with up to 18 years of follow up. Acta Diabetol. 55, 1037-1042. doi: 10.1007/ s00592-018-1176-1

Li, L., Zhao, Q., and Kong, W. (2018). Extracellular matrix remodeling and cardiac fibrosis. Matrix Biol. 68-69, 490-506. doi: 10.1016/j.matbio.2018.01.013

Lillycrop, K. A., Phillips, E. S., Jackson, A. A., Hanson, M. A., and Burdge, G. C. (2005). Dietary protein restriction of pregnant rats induces and folic acid supplementation prevents epigenetic modification of hepatic gene expression in the offspring. J. Nutr. 135, 1382-1386. doi: 10.1093/jn/135.6.1382

Lister, R., Zhou, B., Chamberlain, A., and Einstein, F. (2013). Intrauterine programming of diabetes induced cardiac embryopathy. Am. J. Obstet. Gynecol. 208, S36. doi: 10.1016/j.ajog.2012.10.235 
Lohse, Z., Knorr, S., Bytoft, B., Clausen, T. D., Jensen, R. B., Oturai, P., et al. (2018). Differential effects of age and sex on insulin sensitivity and body composition in adolescent offspring of women with type 1 diabetes: results from the EPICOM study. Diabetologia 61, 210-219. doi: 10.1007/ s00125-017-4458-1

Lu, J., Zhang, S., Li, W., Leng, J., Wang, L., Liu, H., et al. (2019). Maternal gestational diabetes is associated with offspring's hypertension. Am. J. Hypertens. 32, 335-342. doi: 10.1093/ajh/hpz005

Manderson, J., Mullan, B., Patterson, C., Hadden, D., Traub, A., and McCance, D. (2002). Cardiovascular and metabolic abnormalities in the offspring of diabetic pregnancy. Diabetologia 45, 991-996. doi: 10.1007/s00125-002-0865-y

McGavock, J. M., Victor, R. G., Unger, R. H., and Szczepaniak, L. S. (2006). Adiposity of the heart, revisited. Ann. Intern. Med. 144, 517-524. doi: 10.7326/0003-4819-144-7-200604040-00011

Mdaki, K. S., Larsen, T. D., Wachal, A. L., Schimelpfenig, M. D., Weaver, L. J., Dooyema, S. D. R., et al. (2016). Maternal high-fat diet impairs cardiac function in offspring of diabetic pregnancy through metabolic stress and mitochondrial dysfunction. Am. J. Physiol. Heart Circ. Physiol. 310, H681H692. doi: 10.1152/ajpheart.00795.2015

Musikant, D., Sato, H., Capobianco, E., White, V., Jawerbaum, A., and Higa, R. (2019). Altered FOXO1 activation in the programming of cardiovascular alterations by maternal diabetes. Mol. Cell. Endocrinol. 479, 78-86. doi: 10.1016/j.mce.2018.09.003

Najafi, F., Hasani, J., Izadi, N., Hashemi-Nazari, S.-S., Namvar, Z., Mohammadi, S., et al. (2019). The effect of prepregnancy body mass index on the risk of gestational diabetes mellitus: a systematic review and dose-response metaanalysis. Obes. Rev. 20, 472-486. doi: 10.1111/obr.12803

Nasu-Kawaharada, R., Nakamura, A., Kakarla, S. K., Blough, E. R., Kohama, K., and Kohama, T. (2013). A maternal diet rich in fish oil may improve cardiac Akt-related signaling in the offspring of diabetic mother rats. Nutrition 29, 688-692. doi: 10.1016/j.nut.2012.11.017

Pandey, A., Chawla, S., and Guchhait, P. (2015). Type-2 diabetes: current understanding and future perspectives. IUBMB Life 67, 506-513. doi: 10.1002/ iub. 1396

Pathirana, M. M., Lassi, Z. S., Roberts, C. T., and Andraweera, P. H. (2019). Cardiovascular risk factors in offspring exposed to gestational diabetes mellitus in utero: systematic review and meta-analysis. J. Dev. Orig. Health Dis. 11, 599-616. doi: 10.1017/S2040174419000850

Peoples, J. N., Saraf, A., Ghazal, N., Pham, T. T., and Kwong, J. Q. (2019). Mitochondrial dysfunction and oxidative stress in heart disease. Exp. Mol. Med. 51, 1-13. doi: 10.1038/s12276-019-0355-7

Pitzalis, M. V., Sarzani, R., Dessì-Fulgheri, P., Iacoviello, M., Forleo, C., Lucarelli, K., et al. (2003). Allelic variants of natriuretic peptide receptor genes are associated with family history of hypertension and cardiovascular phenotype. J. Hypertens. 21, 1491-1496. doi: 10.1097/00004872-200308000-00012

Planavila, A., Dominguez, E., Navarro, M., Vinciguerra, M., Iglesias, R., Giralt, M., et al. (2012). Dilated cardiomyopathy and mitochondrial dysfunction in Sirt1-deficient mice: a role for Sirt1-Mef2 in adult heart. J. Mol. Cell. Cardiol. 53, 521-531. doi: 10.1016/j.yjmcc.2012.07.019

Poston, L. (2010). Developmental programming and diabetes - the human experience and insight from animal models. Best Pract. Res. Clin. Endocrinol. Metab. 24, 541-552. doi: 10.1016/j.beem.2010.05.007

Preston, C. C., Larsen, T. D., Eclov, J. A., Louwagie, E. J., Gandy, T. C. T., Faustino, R. S., et al. (2020). Maternal high fat diet and diabetes disrupts transcriptomic pathways that regulate cardiac metabolism and cell fate in newborn rat hearts. Front. Endocrinol. 11:570846. doi: 10.3389/ fendo.2020.570846

Regitz-Zagrosek, V., and Kararigas, G. (2017). Mechanistic pathways of sex differences in cardiovascular disease. Physiol. Rev. 97, 1-37. doi: 10.1152/ physrev.00021.2015

Roberti, S. L., Higa, R., Sato, H., Gomez Ribot, D., Capobianco, E., and Jawerbaum, A. (2020). Olive oil supplementation prevents extracellular matrix deposition and reduces prooxidant markers and apoptosis in the offspring's heart of diabetic rats. Reprod. Toxicol. 95, 137-147. doi: 10.1016/j. reprotox.2020.05.002

Rueda-Clausen, C. F., Morton, J. S., Oudit, G. Y., Kassiri, Z., Jiang, Y., and Davidge, S. T. (2012). Effects of hypoxia-induced intrauterine growth restriction on cardiac siderosis and oxidative stress. J. Dev. Orig. Health Dis. 3, 350-357. doi: $10.1017 /$ S2040174412000219
Saxton, R. A., and Sabatini, D. M. (2017). mTOR signaling in growth, metabolism, and disease. Cell 168, 960-976. doi: 10.1016/j.cell.2017.02.004

Sheller-Miller, S., Choi, K., Choi, C., and Menon, R. (2019). Cyclic-recombinasereporter mouse model to determine exosome communication and function during pregnancy. Am. J. Obstet. Gynecol. 221, 502.e1-502.e12. doi: 10.1016/j. ajog.2019.06.010

Shi, R., Zhao, L., Cai, W., Wei, M., Zhou, X., Yang, G., et al. (2017). Maternal exosomes in diabetes contribute to the cardiac development deficiency. Biochem. Biophys. Res. Commun. 483, 602-608. doi: 10.1016/j.bbrc.2016.12.097

Silva, L., Plösch, T., Toledo, F., Faas, M. M., and Sobrevia, L. (2020). Adenosine kinase and cardiovascular fetal programming in gestational diabetes mellitus. Biochim. Biophys. Acta Mol. Basis Dis. 1866:165397. doi: 10.1016/j. bbadis.2019.01.023

Smoak, I. W. (2004). Hyperglycemia-induced TGFbeta and fibronectin expression in embryonic mouse heart. Dev. Dyn. 231, 179-189. doi: 10.1002/dvdy.20123

Tong, M., Chen, Q., James, J. L., Wise, M. R., Stone, P. R., and Chamley, L. W. (2017). In vivo targets of human placental micro-vesicles vary with exposure time and pregnancy. Reproduction 153, 835-845. doi: 10.1530/REP-16-0615

Trujillo, J., Vigo, A., Duncan, B. B., Falavigna, M., Wendland, E. M., Campos, M. A., et al. (2015). Impact of the international association of diabetes and pregnancy study groups criteria for gestational diabetes. Diabetes Res. Clin. Pract. 108, 288-295. doi: 10.1016/j.diabres.2015.02.007

Tuleta, I., and Frangogiannis, N. G. (2021). Diabetic fibrosis. Biochim. Biophys. Acta Mol. Basis Dis. 1867:166044. doi: 10.1016/j.bbadis.2020.166044

Upadhyaya, B., Larsen, T., Barwari, S., Louwagie, E. J., Baack, M. L., and Dey, M. (2017). Prenatal exposure to a maternal high-fat diet affects histone modification of cardiometabolic genes in newborn rats. Nutrients 9:407. doi: 10.3390/nu9040407

van Rijen, H. V., van Veen, T. A., Gros, D., Wilders, R., and de Bakker, J. M. (2006). Connexins and cardiac arrhythmias. Adv. Cardiol. 42, 150-160. doi: $10.1159 / 000092567$

Vijay, V., Han, T., Moland, C. L., Kwekel, J. C., Fuscoe, J. C., and Desai, V. G. (2015). Sexual dimorphism in the expression of mitochondria-related genes in rat heart at different ages. PLoS One 10:e0117047. doi: 10.1371/journal. pone.0117047

Vivar, R., Anfossi, R., Humeres, C., Catalán, M., Reyes, C., Cárdenas, S., et al. (2021). FoxO1 is required for high glucose-dependent cardiac fibroblasts into myofibroblast phenoconversion. Cell. Signal. 83, 109978. doi: 10.1016/j. cellsig.2021.109978

Watson, C. N., Belli, A., and Di Pietro, V. (2019). Small non-coding RNAs: new class of biomarkers and potential therapeutic targets in neurodegenerative disease. Front. Genet. 10:364. doi: 10.3389/fgene.2019.00364

Wende, A. R., and Abel, E. D. (2010). Lipotoxicity in the heart. Biochim. Biophys. Acta 1801, 311-319. doi: 10.1016/j.bbalip.2009.09.023

West, N. A., Crume, T. L., Maligie, M. A., and Dabelea, D. (2011). Cardiovascular risk factors in children exposed to maternal diabetes in utero. Diabetologia 54, 504-507. doi: 10.1007/s00125-010-2008-1

West, N. A., Kechris, K., and Dabelea, D. (2013). Exposure to maternal diabetes in utero and DNA methylation patterns in the offspring. Immunometabolism 1, 1-9. doi: 10.2478/immun-2013-0001

Westermeier, F., Salomón, C., Farías, M., Arroyo, P., Fuenzalida, B., Sáez, T., et al. (2015). Insulin requires normal expression and signaling of insulin receptor $\mathrm{A}$ to reverse gestational diabetes-reduced adenosine transport in human umbilical vein endothelium. FASEB J. 29, 37-49. doi: 10.1096/ f.14-254219

Westermeier, F., Salomón, C., González, M., Puebla, C., Guzmán-Gutiérrez, E., Cifuentes, F., et al. (2011). Insulin restores gestational diabetes mellitusreduced adenosine transport involving differential expression of insulin receptor isoforms in human umbilical vein endothelium. Diabetes 60, 1677-1687. doi: 10.2337/db11-0155

Xu, H. E., Lambert, M. H., Montana, V. G., Parks, D. J., Blanchard, S. G., Brown, P. J., et al. (1999). Molecular recognition of fatty acids by peroxisome proliferator-activated receptors. Mol. Cell 3, 397-403. doi: 10.1016/ S1097-2765(00)80467-0

Yin, Y., and Yang, C. (2019). miRNA-30-3p improves myocardial ischemia via the PTEN/PI3K/AKT signaling pathway. J. Cell. Biochem. 120, 17326-17336. doi: $10.1002 /$ jcb. 28996

Yu, Y., Arah, O. A., Liew, Z., Cnattingius, S., Olsen, J., Sorensen, H. T., et al. (2019). Maternal diabetes during pregnancy and early onset of cardiovascular 
disease in offspring: population based cohort study with 40 years of followup. $B M J$ 367:16398. doi: $10.1136 / \mathrm{bmj} .16398$

Zhang, J., Cao, L., Tan, Y., Zheng, Y., and Gui, Y. (2021). N-acetylcysteine protects neonatal mice from ventricular hypertrophy induced by maternal obesity in a sex-specific manner. Biomed. Pharmacother. 133:110989. doi: 10.1016/j.biopha.2020.110989

Zhang, L., Wang, X., Wu, Y., Lu, X., Chidiac, P., Wang, G., et al. (2018). Maternal diabetes up-regulates NOX2 and enhances myocardial ischaemia/ reperfusion injury in adult offspring. J. Cell. Mol. Med. 22, 2200-2209. doi: $10.1111 / \mathrm{jcmm} .13500$

Zhou, F. C., Chen, Y., and Love, A. (2011). Cellular DNA methylation program during neurulation and its alteration by alcohol exposure. Birth Defects Res. A Clin. Mol. Teratol. 91, 703-715. doi: 10.1002/bdra.20820

Zhu, Y., and Zhang, C. (2016). Prevalence of gestational diabetes and risk of progression to type 2 diabetes: a global perspective. Curr. Diab. Rep. 16, 7. doi: $10.1007 /$ s11892-015-0699-x
Conflict of Interest: The authors declare that the research was conducted in the absence of any commercial or financial relationships that could be construed as a potential conflict of interest.

Publisher's Note: All claims expressed in this article are solely those of the authors and do not necessarily represent those of their affiliated organizations, or those of the publisher, the editors and the reviewers. Any product that may be evaluated in this article, or claim that may be made by its manufacturer, is not guaranteed or endorsed by the publisher.

Copyright (๑) 2021 Higa, Leonardi and Jawerbaum. This is an open-access article distributed under the terms of the Creative Commons Attribution License (CC BY). The use, distribution or reproduction in other forums is permitted, provided the original author(s) and the copyright owner(s) are credited and that the original publication in this journal is cited, in accordance with accepted academic practice. No use, distribution or reproduction is permitted which does not comply with these terms. 